\title{
TITLE:
}

\section{A Simplified Domain Structure Model Exhibiting the Pinning Field}

$\operatorname{AUTHOR}(\mathrm{S})$ :

Sudo, Masato; Mifune, Takeshi; Matsuo, Tetsuji; Kaido, Chikara

\section{CITATION:}

Sudo, Masato ...[et al]. A Simplified Domain Structure Model Exhibiting the Pinning Field. IEEE Transactions on Magnetics 2013, 49(5): 18291832

ISSUE DATE:

2013-05

URL:

http://hdl.handle.net/2433/174349

\section{RIGHT:}

(c) 2013 IEEE. Personal use of this material is permitted. Permission from IEEE must be obtained for all other uses, in any current or future media, including reprinting/republishing this material for advertising or promotional purposes,

creating new collective works, for resale or redistribution to servers or lists, or reuse of any copyrighted component of this work in other works.; This is not the published version. Please cite only the published version.; この論文は出版社版

でありません。引用の際には出版社版をご確認ご利用ください。 


\title{
A Simplified Domain Structure Model Exhibiting the Pinning Field
}

\author{
Masato Sudo ${ }^{1}$, Takeshi Mifune ${ }^{1}$, Tetsuji Matsuo ${ }^{1}$, Member IEEE, Chikara Kaido ${ }^{2}$ \\ ${ }^{1}$ Graduate School of Engineering, Kyoto University, Kyoto, 615-8510 Japan \\ ${ }^{2}$ Kitakyushu National College of Technology, Kitakyushu, 802-0985 Japan
}

\begin{abstract}
A simplified domain structure model (SDSM) is developed as a building block with which to construct a physical macroscopic magnetization model exhibiting pinning-type hysteresis. The pinning field is represented by a stop hysteron. The proposed SDSM represents the vector magnetic property of a silicon steel sheet qualitatively. A preliminary analysis of the magnetization process described by the assembled SDSMs is reported, where the local demagnetizing field reduces the coercive force.
\end{abstract}

Index Terms—Demagnetizing field, magnetic domain, mesoscopic model, pinning field

\section{INTRODUCTION}

$\mathrm{M}$ acroscopic magnetic properties of iron-core material are governed by mesoscopic magnetization processes on the crystal-grain scale such as domain wall motion and magnetization rotation [1]-[4]. Domain structure models [5][8] have been developed for mesoscopic magnetization analysis and have been applied to the analysis of sheet-type magnetic materials. To describe the basic mechanism of mesoscopic magnetization, a simplified domain structure model (SDSM) [9], [10] has been proposed and has successfully described the properties of silicon steel qualitatively including the rotational magnetic property. However, a single SDSM cannot describe the macroscopic magnetization of realistic iron-core because the SDSM is too simple to handle a complex domain structure. In addition, it is necessary for the SDSM to describe the mesoscopic pinning effect to represent macroscopic pinning-type hysteresis [4].

This paper discusses the assembly of SDSMs to constitute a physical macroscopic magnetization model that represents anisotropic vector magnetic properties of silicon steel. This study also develops an SDSM that exhibits the pinning field using the vector stop hysteron [11] and addresses the preliminary analysis of the magnetization process described by assembled SDSMs.

\section{Simplified DOMAIN STRUCTURE MODEL}

An SDSM [9], [10] with two domains is used to describe the behavior of a mesoscopic magnetic particle, where the magnetization is assumed uniform in each domain $i(i=1,2)$. The normalized magnetization vector in domain $i$ is given by $\boldsymbol{m}_{i}=\left(\sin \theta_{i} \cos \varphi_{i}, \sin \theta_{i} \sin \varphi_{i}, \cos \theta_{i}\right)$.

\section{A. Energy components}

The total magnetic energy to be minimized is normalized by the crystalline anisotropy energy [9]. The normalized total magnetic energy, $e$, is given as

$$
e=e_{\mathrm{ap}}+e_{\mathrm{an}}+e_{\mathrm{w}}+e_{\mathrm{st}}
$$

where $e_{\mathrm{ap}}$ is the Zeeman energy, $e_{\mathrm{an}}$ is the crystalline anisotropic energy, $e_{\mathrm{w}}$ is the domain-wall energy, and $e_{\mathrm{st}}$ is the magnetostatic energy. The normalized crystalline anisotropic

Manuscript received November 10, 2012 (date on which paper was submitted for review). Corresponding author: T. Matsuo (e-mail: tmatsuo@kuee.kyoto-u.ac.jp).

Digital Object Identifier inserted by IEEE energy is given as

$$
e_{\text {an }}=\lambda f_{\text {an }}\left(\theta_{1}, \varphi_{1}\right)+(1-\lambda) f_{\text {an }}\left(\theta_{2}, \varphi_{2}\right)
$$

where $f_{\text {an }}$ represents the angular dependence and $\lambda$ is the volume ratio of domain 1 . The Zeeman energy due to the normalized applied field, $\boldsymbol{h}=h\left(\cos \varphi_{\mathrm{H}}, \sin \varphi_{\mathrm{H}}, 0\right)$, is given as $e_{\text {ap }}=-2 \boldsymbol{h} \cdot\left[\lambda \boldsymbol{m}_{1}+(1-\lambda) \boldsymbol{m}_{2}\right]$, where $h=H_{\mathrm{ap}} /\left(\kappa M_{\mathrm{S}}\right), H_{\mathrm{ap}}$ is the magnitude of the applied magnetic field, $M_{\mathrm{S}}$ is the magnitude of spontaneous magnetization, $\kappa=2 K /\left(\mu_{0} M_{\mathrm{S}}{ }^{2}\right)$ and $K$ is the anisotropy constant. A simple Bloch wall model gives the domain-wall energy as $e_{\mathrm{w}}=w\left(1-\boldsymbol{m}_{1} \cdot \boldsymbol{m}_{2}\right) / 2$, where $w=4 l_{\mathrm{k}} / D$, $l_{\mathrm{k}}=(A / K)^{1 / 2}$ and $D$ is the width of the two domains. The parameter, $w$, represents the energy cost of a domain wall. The SDSM assumes that the demagnetizing field is uniformly given by the multiplication of demagnetizing factors and the average magnetization. The magnetostatic energy is given as

$$
\begin{aligned}
& e_{\mathrm{st}}=s_{x} m_{x}^{2}+s_{y} m_{y}^{2}+s_{z} m_{z}^{2} \\
& \left(m_{x}, m_{y}, m_{z}\right)=\boldsymbol{m}=\lambda \boldsymbol{m}_{1}+(1-\lambda) \boldsymbol{m}_{2}
\end{aligned}
$$

where $s_{x}=k_{x} / \kappa, s_{y}=k_{y} / \kappa$ and $s_{z}=k_{z} / \kappa ; k_{x}, k_{y}$, and $k_{z}$ are the demagnetizing factors.

\section{B. Local energy minimization}

The magnetization is determined by finding a local energy minimum that satisfies $\partial e / \partial \boldsymbol{X}=0$, where $\boldsymbol{X}=\left(\theta_{1}, \varphi_{1}, \theta_{2}, \varphi_{2}, \lambda\right)$.

The total energy $e$ often has several local minima, one of which is chosen according to the magnetization history. To represent the dependence on the history, it is proposed in this paper to find an equilibrium point of artificial state equations given as

$$
\mathrm{d} \boldsymbol{X} / \mathrm{d} t=\boldsymbol{Y}, \mathrm{d} \boldsymbol{Y} / \mathrm{d} t=-\partial e / \partial \boldsymbol{X}-\alpha \boldsymbol{Y}
$$

where $\boldsymbol{Y}$ is an intermediate variable vector and $\alpha$ is a dissipation coefficient promoting a fast convergence to an equilibrium point. A local energy minimum depending on the initial condition is given by the numerical integration of (5), obtaining the steady state where $\mathrm{d} \boldsymbol{X} / \mathrm{d} t=\mathrm{d} \boldsymbol{Y} / \mathrm{d} t=0$.

\section{PINNING EFFECT}

Soft magnetic materials often exhibit pinning-type hysteresis with gradual domain-wall motion. The pinning-type hysteresis is mainly caused by the interaction of domain walls with crystal defects and crystal grain boundaries.

When the distribution of pinning sites and the magnitude of 
pinning energy are assumed uniform, there is a uniform pinning field, whose direction depends on the direction of $\mathrm{d} \boldsymbol{m} / \mathrm{d} t$. Accordingly, a uniform pinning field $h_{\mathrm{p}}$ is described by a stop hysteron [11] under the alternating field as

$$
\begin{aligned}
& h_{\mathrm{pn}}=-\partial e_{\mathrm{pn}} / \partial m=-(p / \eta) s_{\eta}(m) \\
& s_{\eta}(m)=\max \left(\min \left(m-m^{0}+s_{\eta}^{0}, \eta\right),-\eta\right)
\end{aligned}
$$

where $s_{\eta}$ is the stop hysteron with height $\eta,\left(m^{0}, s_{\eta}{ }^{0}\right)$ are previous values of $\left(m, s_{\eta}\right)$, and $p$ is a constant that gives the magnitude of the pinning field. Fig. 1 illustrates the property of $(p / \eta) s_{\eta}(m)$. When the magnetization is increasing (or decreasing), an additional applied field of $p$ (or $-p)$ is required to compensate the pinning field $h_{\mathrm{pn}}=-p$ (or $p$ ).

The pinning energy for the vector field is given by

$$
e_{\mathrm{pn}}=\frac{p}{\eta} \int \boldsymbol{s}_{\eta}(\boldsymbol{m}) \cdot \mathrm{d} \boldsymbol{m}
$$

where $\boldsymbol{s}_{\eta}(\boldsymbol{m})$ is a vector stop hysteron; $\boldsymbol{s}_{\eta}(\boldsymbol{m})$ is given as

$$
\boldsymbol{s}_{\eta}(\boldsymbol{m})=\frac{\eta\left(\boldsymbol{m}-\boldsymbol{m}^{0}+\boldsymbol{s}_{\eta}{ }^{0}\right)}{\max \left(\eta,\left|\boldsymbol{m}-\boldsymbol{m}^{0}+\boldsymbol{s}_{\eta}{ }^{0}\right|\right)}
$$

where $\eta$ is a constant giving the radius of the stop hysteron, and $\left(\boldsymbol{m}^{0}, \boldsymbol{s}_{\eta}{ }^{0}\right)$ are the values of $\left(\boldsymbol{m}, \boldsymbol{s}_{\eta}\right)$ at the previous timepoint. For simplicity, the pinning energy is assumed to be independent of the other energy components. Accordingly, the pinning energy $e_{\mathrm{pn}}$ is simply added to the total energy $e$.

Similar to the case for a grain-oriented silicon steel sheet [10], cubic crystalline anisotropy is assumed in this paper, where the three axes of easy magnetization are set along the (1, $0,0),(0,1,1)$, and $(0,-1,1)$ directions. Thus, the anisotropic function $f_{\mathrm{an}}\left(\theta_{i}, \varphi_{i}\right)$ is given as

$$
\begin{aligned}
& f_{\text {an }}\left(\theta_{i}, \varphi_{i}\right) \\
& =\sin ^{2} \theta_{i} \cos ^{2} \varphi_{i}\left(1-\cos ^{2} \varphi_{i} \sin ^{2} \theta_{i}\right)+\frac{1}{4}\left(\cos ^{2} \theta_{i}-\sin ^{2} \theta_{i} \sin ^{2} \varphi_{i}\right)^{2}
\end{aligned}
$$

where the $x$ - and $y$-directions correspond to the rolling direction (RD) and transverse direction (TD), respectively.

Fig. 2 shows magnetization curves when $w=0.01, s_{x}=0.5$, $s_{y}=5$, and $s_{z}=100$ in the cases (a) without $e_{\mathrm{pn}}(p=0)$, (b) with $p=1$ and $\eta=0.1$, (c) with $p=1$ and $\eta=0.01$, and (d) with $p=$ 2 and $\eta=0.1$. Assuming a sheet structure, $s_{z}$ is very large compared with $s_{x}$ and $s_{y}$. The magnetization curves in Fig. 2(a) coincide with those obtained in [10] where $\partial e / \partial \boldsymbol{X}=0$ is solved directly. The pinning field increases the coercive force along the RD and yields hysteresis along the TD. Fig. 3 portrays the measured magnetization curves of a grain-oriented silicon steel sheet. The sharp increase in the magnetization with a nearly rectangular hysteresis loop along the $\mathrm{RD}$ is represented by the SDSM. The complex magnetization curve measured along the TD is a result of the transition of the magnetization state [1], [2], which is represented by the SDSM qualitatively. However, the coercive force of steel sheet along the RD is rather small compared with the anisotropic field that determines the coercive force in the SDSM [9]. The magnetization state transition along the TD does not clearly affect the MH curve simulated by the SDSM because the effect of the demagnetizing field is dominant in the simulated SDSM. The above property difference is due to the property of silicon steel being governed by the assembly of crystal grains. The following section examines an assembly of SDSMs used to construct a macroscopic model where the SDSM is used as a building block.

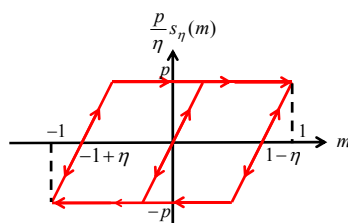

Fig. 1. Property of the stop hysteron
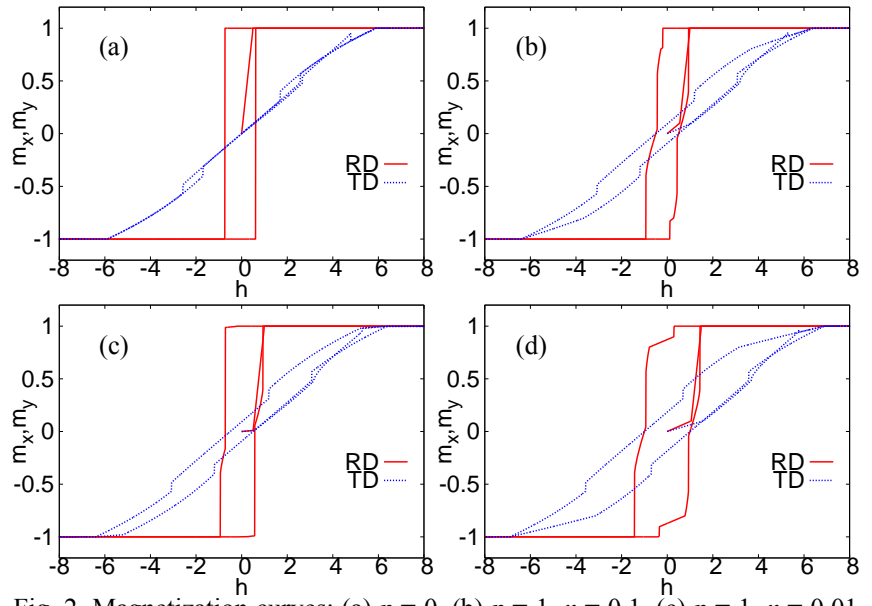

Fig. 2. Magnetization curves: (a) $p=0$, (b) $p=1, \eta=0.1$, (c) $p=1, \eta=0.01$, and (d) $p=2, \eta=0.1$.

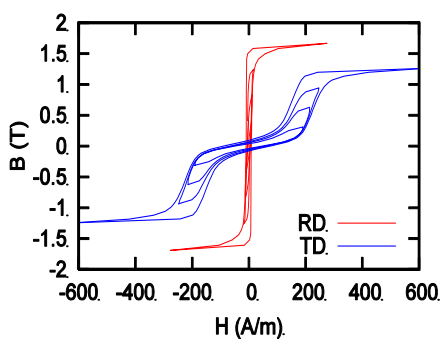

Fig. 3. Measured magnetization curves of a grain-oriented silicon steel sheet along the rolling direction (RD) and transverse direction (TD).

\section{ASSEMBLY OF SDSMs}

A single SDSM cannot describe the macroscopic magnetization of realistic iron-core material because of its simplicity. To construct a macroscopic magnetization model, an assembly of SDSMs is examined. Fig. 4 illustrates an assembly of SDSMs, where each SDSM is regarded as a cell constituting the macroscopic model.

The total magnetic energy, $e$, is given as

$$
e=e_{\text {ap-global }}+e_{\text {an-global }}+e_{\mathrm{w} \text {-global }}+e_{\text {pn-global }}+e_{\text {st-global }}
$$

where each of the first four components on the right-hand side is given by the summation of the local components $e_{\mathrm{ap}}, e_{\mathrm{an}}, e_{\mathrm{w}}$ and $e_{\mathrm{pn}}$ in each cell without interaction between the cells. The normalized magnetostatic energy $e_{\text {st-global }}$ is computed as described in the following subsection. 


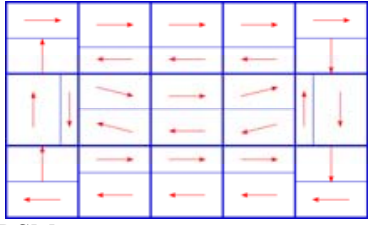

Fig. 4. Assembly of SDSMs.

\section{A. Demagnetizing field}

The demagnetizing field $\boldsymbol{H}_{\mathrm{st}}$ in the assembly model is obtained in the same way as in micromagnetic simulation [12]; $\boldsymbol{H}_{\mathrm{st}}$ is given as

$$
\boldsymbol{H}_{\mathrm{st}}(I, J, K)=-M_{s} \sum_{I^{\prime}, J^{\prime}, K^{\prime}} \boldsymbol{N}\left(I-I^{\prime}, J-J^{\prime}, K-K^{\prime}\right) \boldsymbol{m}\left(I^{\prime}, J^{\prime}, K^{\prime}\right)
$$

where $(I, J, K)$ and $\left(I^{\prime}, J^{\prime}, K^{\prime}\right)$ are cell index numbers. The demagnetizing coefficient matrix $N=\left\{N_{u v}\right\}$ is given as

$$
N_{u v}(I, J, K)=\frac{1}{4 \pi} \int_{\left(K-\frac{1}{2}\right) \Delta z}^{\left(K+\frac{1}{2}\right) \Delta z\left(J-\frac{1}{2}\right) \Delta y\left(I-\frac{1}{2}\right) \Delta y} \int_{\left.\frac{1}{2}\right) \Delta x}^{\left(I+\frac{1}{2}\right) \Delta x} \frac{\delta_{u v}\left(x^{2}+y^{2}+z^{2}\right)-3 u v}{\left(x^{2}+y^{2}+z^{2}\right)^{5 / 2}} \mathrm{~d} x \mathrm{~d} y \mathrm{~d} z
$$

where $u$ and $v$ are $x, y$ or $z$; $\delta_{u v}$ denotes Kronecker's delta, and $(\Delta x, \Delta y, \Delta z)$ is the cell size. For simplicity of notation, the cell index is renumbered as

$$
\begin{aligned}
& i=n_{x} n_{y}(K-1)+n_{y}(J-1)+I \\
& \left(I=1, \ldots, n_{x}, J=1, \ldots, n_{y}, K=1, \ldots, n_{z}\right)
\end{aligned}
$$

where $n_{x}, n_{y}$, and $n_{z}$ are the numbers of cells along the $x$-, $y$ and $z$-directions, respectively. Using the index $i$ above, the magnetostatic energy is given as

$$
E_{\mathrm{st}}=-\frac{\mu_{0} M_{s} V}{2} \sum_{i} \boldsymbol{H}_{\mathrm{st}}(i) \cdot \boldsymbol{m}(i)
$$

where $\boldsymbol{H}_{\mathrm{st}}(i)$ and $\boldsymbol{m}(i)$ are the demagnetizing field and the average magnetization at cell $i$, and $V$ is the cell volume. The normalized magnetostatic energy is given by

$$
e_{\text {st-global }}=E_{\mathrm{st}} / V K=-\sum_{i} \boldsymbol{h}_{\mathrm{st}}(i) \cdot \boldsymbol{m}(i)
$$

where $\boldsymbol{h}_{\mathrm{st}}$ is the normalized demagnetizing field; $\boldsymbol{h}_{\mathrm{st}}$ is given as

$$
\boldsymbol{h}_{\mathrm{st}}(i)=\boldsymbol{H}_{\mathrm{st}} /\left(\kappa M_{\mathrm{S}}\right)=-\sum_{i^{\prime}} \boldsymbol{s}\left(i-i^{\prime}\right) \boldsymbol{m}\left(i^{\prime}\right)
$$

where

$$
\boldsymbol{s}(i)=N(I, J, K) / \kappa .
$$

From (19), (20) and $\boldsymbol{s}(i)=\boldsymbol{s}(-i), \partial e_{\text {st-global }} / \partial \boldsymbol{m}(j)$ is given as

$$
\begin{aligned}
\frac{\partial \boldsymbol{e}_{\text {st-global }}}{\partial \boldsymbol{m}(j)} & =-\boldsymbol{h}_{\mathrm{st}}(j)^{\mathrm{T}}-\sum_{i} \boldsymbol{m}(i)^{\mathrm{T}} \frac{\partial \boldsymbol{h}_{\mathrm{st}}(i)}{\partial \boldsymbol{m}(j)} \\
& =-\boldsymbol{h}_{\mathrm{st}}(j)^{\mathrm{T}}-\sum_{i} \boldsymbol{m}(i)^{\mathrm{T}} \boldsymbol{s}(j-i)=-2 \boldsymbol{h}_{\mathrm{st}}(j)^{\mathrm{T}}
\end{aligned}
$$

\section{B. Local energy minimization}

The state variable vector $\boldsymbol{X}$ consists of $\boldsymbol{X}(i)(i=1, \ldots$, $\left.n_{x} n_{y} n_{z}\right)$ in each cell. A local energy minimum point is obtained by solving (5), where $\partial e_{\text {st-global }} / \partial \boldsymbol{X}(i)$ is given as

$$
\frac{\partial e_{\text {st-global }}}{\partial \boldsymbol{X}(i)}=\frac{\partial e_{\text {st-global }}}{\partial \boldsymbol{m}(i)} \frac{\partial \boldsymbol{m}(i)}{\partial \boldsymbol{X}(i)}=-2 \boldsymbol{h}_{\mathrm{st}}(i)^{\mathrm{T}} \frac{\partial \boldsymbol{m}(i)}{\partial \boldsymbol{X}(i)} .
$$

The differentiation of the other component is straightforward.

\section{Preliminary Analysis USIng an AsSembly Model}

Since the SDSM assumes a uniform demagnetizing field, it cannot take account of the effect of the material edge. However, magnetization reversal often begins at the material edge. This section carries out a preliminary magnetization process analysis using the assembly model of small number of DSMSs to examine the effect of the local demagnetizing field.

\section{A. Effect of the division into multi-SDSMs}

A magnetic material having dimensions $l_{x} \times l_{y} \times l_{z}$ is analyzed with the assembly model, where $l_{x}: l_{y}: l_{z}$ is set to $8: 1: 0.02$. Crystalline anisotropy of (12) is assumed. The material is divided into (i) $1 \times 1 \times 1$ cell, (ii) $2 \times 1 \times 1$ cells, and (iii) $4 \times 1 \times 1$ cells as shown in Fig. 5. Fig. 6(a), (b) and (c) portrays the magnetization curve along the RD and TD without the pinning field, where $w=0.01$ and $\kappa=0.001$.

Fig. 6 shows that the cell division reduces the coercive force along the RD. Table I lists the demagnetizing coefficients;

$$
\boldsymbol{s}(0)=\operatorname{diag}\left(s_{x x}, s_{y y}, s_{z z}\right)
$$

Model (i) has small $s_{x x}\left(<<s_{y y}\right)$, which means that there is large shape anisotropy with the easy axis along the RD producing a large coercive force. Fig. 7 shows the magnetization process for four cells in model (iii). Model (iii) has relatively large $s_{x x}$, which means that the shape anisotropy of a single cell is not large. Consequently, the magnetization in cells 1 and 4 reverses easily because the cells at the material edge have smaller interaction with other cells than the center cells 2 and 3. The magnetization reversal in cells 1 and 4 assists the reversal in cells 2 and 3 , which results in a small coercive force. The cell division along the RD does not affect the magnetization along the TD because $s_{y y}$ is not greatly affected. Fig. 6(d) shows the magnetization property with the pinning field where $p=0.5$ and $\eta=0.1$.
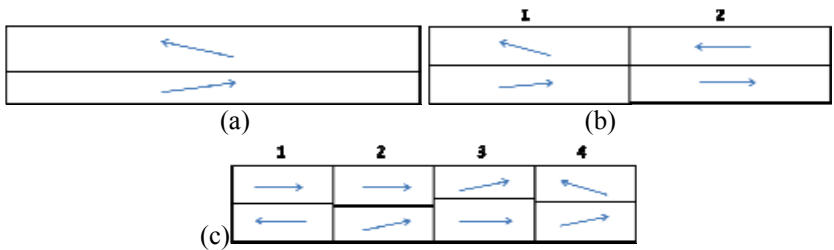

Fig. 5. Assembly model: (a) $1 \times 1 \times 1$ cell, (b) $2 \times 1 \times 1$ cells, and (c) $4 \times 1 \times 1$ cells.

\section{B. Effect of anisotropic energy and magnetostatic energy}

The parameter $\kappa$ represents the ratio of influence of crystalline anisotropy energy to that of magnetostatic energy. Fig. 8 shows the magnetization curves given by model (iii) with $\kappa=0.002,0.01$ and with pinning field of $p=0.5$ and $\eta=$ 0.1. Table II lists the corresponding demagnetizing coefficients. The coercive force along the RD increases with $\kappa$ because of the increase in the strength of the anisotropy field. The susceptibility along the TD increases with $\kappa$ because of the decrease in the strength of the demagnetizing field. The comparison of Fig. 8(a) with Fig. 2 shows that the assembly 
model has smaller coercive force than the single SDSM because of the local demagnetizing field and improves the representation of the properties of grain-oriented silicon steel.

TABLE I.

RELATION BETWEEN CELL DIVISION AND DEMAGNETIZING COEFFICIENTS

\begin{tabular}{c|c|c|c}
\hline$\left(n_{x}, n_{y}, n_{z}\right)$ & $s_{x x}$ & $s_{y y}$ & $S_{z z}$ \\
\hline$(1,1,1):$ model (i) & $2.1 \times 10^{-1}$ & $1.3 \times 10^{1}$ & $1.0 \times 10^{3}$ \\
\hline$(2,1,1):$ model (ii) & $8.2 \times 10^{-1}$ & $1.3 \times 10^{1}$ & $1.0 \times 10^{3}$ \\
\hline$(4,1,1):$ model (iii) & 3.0 & $1.2 \times 10^{1}$ & $1.0 \times 10^{3}$ \\
\hline
\end{tabular}
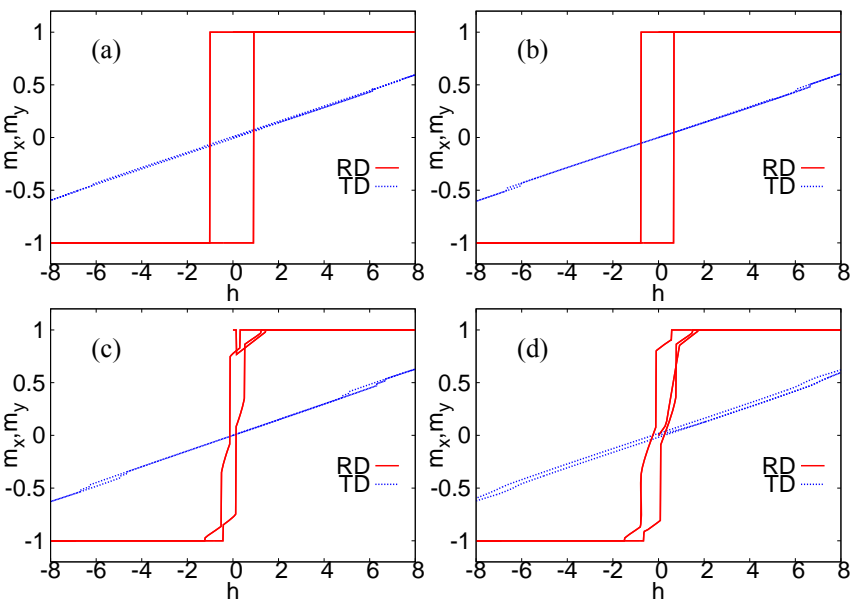

Fig. 6. Magnetization curves: (a) $1 \times 1 \times 1$ cell, (b) $2 \times 1 \times 1$ cells, and (c) $4 \times 1 \times 1$ cells, and (d) $4 \times 1 \times 1$ cells with the pinning field.

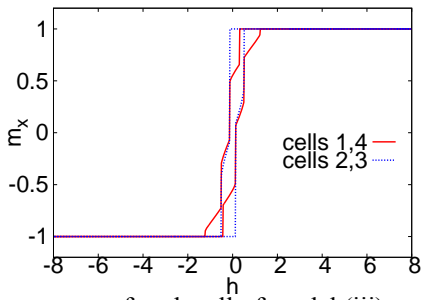

Fig. 7. Magnetization curves of each cell of model (iii)
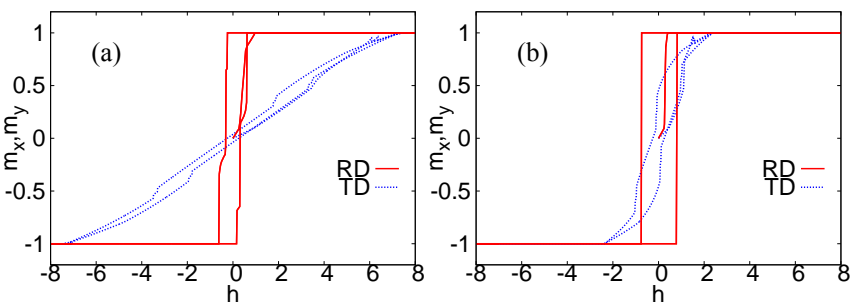

Fig. 8. Magnetization curves obtained with $4 \times 1 \times 1$ cells and the pinning field: (a) $\kappa=0.002$ and (b) $\kappa=0.01$.

TABLE II.

RELATION BETWEEN K AND DEMAGNETIZING COEFFICIENTS

\begin{tabular}{c|c|c|c}
\hline$\kappa$ & $S_{x x}$ & $S_{y y}$ & $S_{z z}$ \\
\hline 0.001 & 3.0 & $1.2 \times 10^{1}$ & $1.0 \times 10^{3}$ \\
\hline 0.002 & 1.4 & 5.9 & $4.9 \times 10^{2}$ \\
\hline 0.01 & $2.8 \times 10^{-1}$ & 1.1 & $9.8 \times 10^{1}$ \\
\hline
\end{tabular}

Fig. 9 compares the MH curves given by the micromagnetic simulation (MMS) and the assembled SDSMs of $4 \times 1 \times 1$ cells without the pinning field. The parameters for the MMS are chosen so as to satisfy $w=0.01, \kappa=0.01$ and $l_{x}: l_{y}: l_{z}=$ $8: 1: 0.02$. The MH curve given by the assembled SDSMs roughly agrees with that yielded by the MMS. The discrepancy between the MH curves is probably caused by the insufficient number of cell divisions that are $4 \times 1 \times 1$ and 256 $\times 32 \times 4$ for the assembled SDSMs and the MMS, respectively.
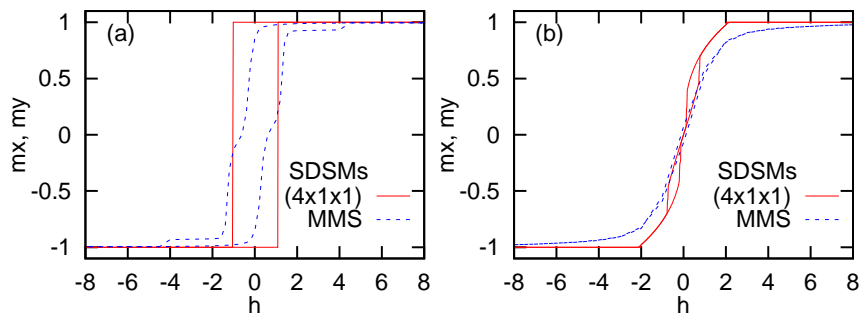

Fig. 9. Magnetization curves obtained by the MMS and assembled SDSMs where $\kappa=0.01, w=0.01$ and $l_{x}: l_{y}: l_{z}=8: 1: 0.02$.

\section{ACKNOWLEDGEMENTS}

This work was supported in part by the JFE 21st Century Foundation through a Technical Research Aid and by the Japan Society for the Promotion of Science through a Grantin-Aid for Scientific Research (C), Grant No.23560328.

\section{REFERENCES}

[1] F. Fiorillo, L. R. Dupre, C. Appino, and A. M. Rietto, "Comprehensive model of magnetization curve, hysteresis loops, and losses in any direction in grain-oriented Fe-Si," IEEE Trans. Magn., vol. 38, pp. 1467-1476, May 2002.

[2] O. Hubert, L. Daniel, "Multiscale modeling of the magneto-mechanical behavior of grain-oriented silicon steels," J. Magn. Magn. Mater., vol. 320, pp. 1412-1422, 2008.

[3] A. Hubert, R. Schäfer, Magnetic Domains: The Analysis of Magnetic Microstructures, Springer-Verlag, Berlin Heidelberg, 1998.

[4] G. Bertotti, Hysteresis in Magnetism, Academic Press, 1998,

[5] N. Smith, "Domain theory model for magnetic thin films," IEEE Trans. Magn., vol. 24, pp. 2380-2382, Nov. 1988.

[6] C. Saka, K. Shiiki, and K. Shinagawa, "Simulation of domain structure for magnetic thin film in an applied field," J. Appl. Phys., vol. 68, pp. 263-268, July 1990.

[7] M. Enokizono, T. Todaka, and Y. Midou, "Numerical simulation of domain structure in magnetic thin sheet," IEEE Trans. Magn., vol. 32, pp. 1172-1175, May 1996.

[8] T. Matsuo, N. Mimuro, and M. Shimasaki, "A micromagnetic study of domain structure modeling”, J. Magn. Magn. Mater., vol. 320, pp. e1029-e1033, 2008.

[9] T. Matsuo, "Magnetization process analysis using a simplified domain structure model," J. Appl. Phys., vol. 109, 07D332, 2011.

[10] M. Sudo, T. Matsuo, "Magnetization modeling of silicon steel using a simplified domain structure model," J. Appl. Phys., vol. 111, 07D107, 2012.

[11] T. Matsuo, "Rotational saturation properties of isotropic vector hysteresis models using vectorized stop and play hysterons," IEEE Trans. Magn., vol. 44, pp. 3185-3188, Nov. 2008.

[12] N. Hayasi, K. Saito and Y. Nakatani, "Calculation of demagnetizing field distribution based on fast Fourier transform of convolution," Jpn. J. Appl. Phys., vol.35, pp.6065-6073, 1996. 